\title{
CORRECTION
}

\section{Correction to: Bound smoothing based time series anomaly detection using multiple similarity measures}

\author{
Wenqing Wang ${ }^{1} \cdot$ Junpeng $\mathrm{Bao}^{1}\left[\mathrm{D} \cdot \mathrm{Tao}^{\mathrm{Li}}{ }^{1}\right.$
}

Published online: 25 September 2020

(c) Springer Science+Business Media, LLC, part of Springer Nature 2020

\section{Correction to: \\ Journal of Intelligent Manufacturing https://doi.org/10.1007/s10845-020-01583-0}

After publication, the authors realized that they failed to z-normalize the subsequences for BFDD, making it perform much worse than a correct implementation, such as detection on xmitdb_1108_2. Please download the correct BFDD code on the webpage "https://www.cs.ucr.edu/ eamonn/MatrixProfile.html".

The authors appreciate Professor Keogh's comments on the shortcomings of our results and his contributions to time series mining and benchmark data sets.

Publisher's Note Springer Nature remains neutral with regard to jurisdictional claims in published maps and institutional affiliations.

The original article can be found online at https://doi.org/10.1007/ s10845-020-01583-0.

Junpeng Bao

baojp@mail.xjtu.edu.cn

Wenqing Wang

wenqingwang@stu.xjtu.edu.cn

Tao Li

lit_cs@163.com

1 Department of Computer Science and Technology, Xi' an Jiaotong University, Xi'an, People's Republic of China 